This item was submitted to Loughborough's Research Repository by the author.

Items in Figshare are protected by copyright, with all rights reserved, unless otherwise indicated.

\title{
Microneedles from fish scale biopolymer
}

PLEASE CITE THE PUBLISHED VERSION

http://dx.doi.org/10.1002/app.40377

\section{PUBLISHER}

(c) Wiley Periodicals, Inc.

\section{VERSION}

AM (Accepted Manuscript)

\section{LICENCE}

CC BY-NC-ND 4.0

\section{REPOSITORY RECORD}

Olatunji, Ololade, Chima C. Igwe, Aroke S. Ahmed, Dewale O.A. Alhassan, Godfrey O. Asieba, and Diganta Bhusan Das. 2014. "Microneedles from Fish Scale Biopolymer". figshare. https://hdl.handle.net/2134/14364. 
This item was submitted to Loughborough's Institutional Repository (https://dspace.lboro.ac.uk/) by the author and is made available under the following Creative Commons Licence conditions.

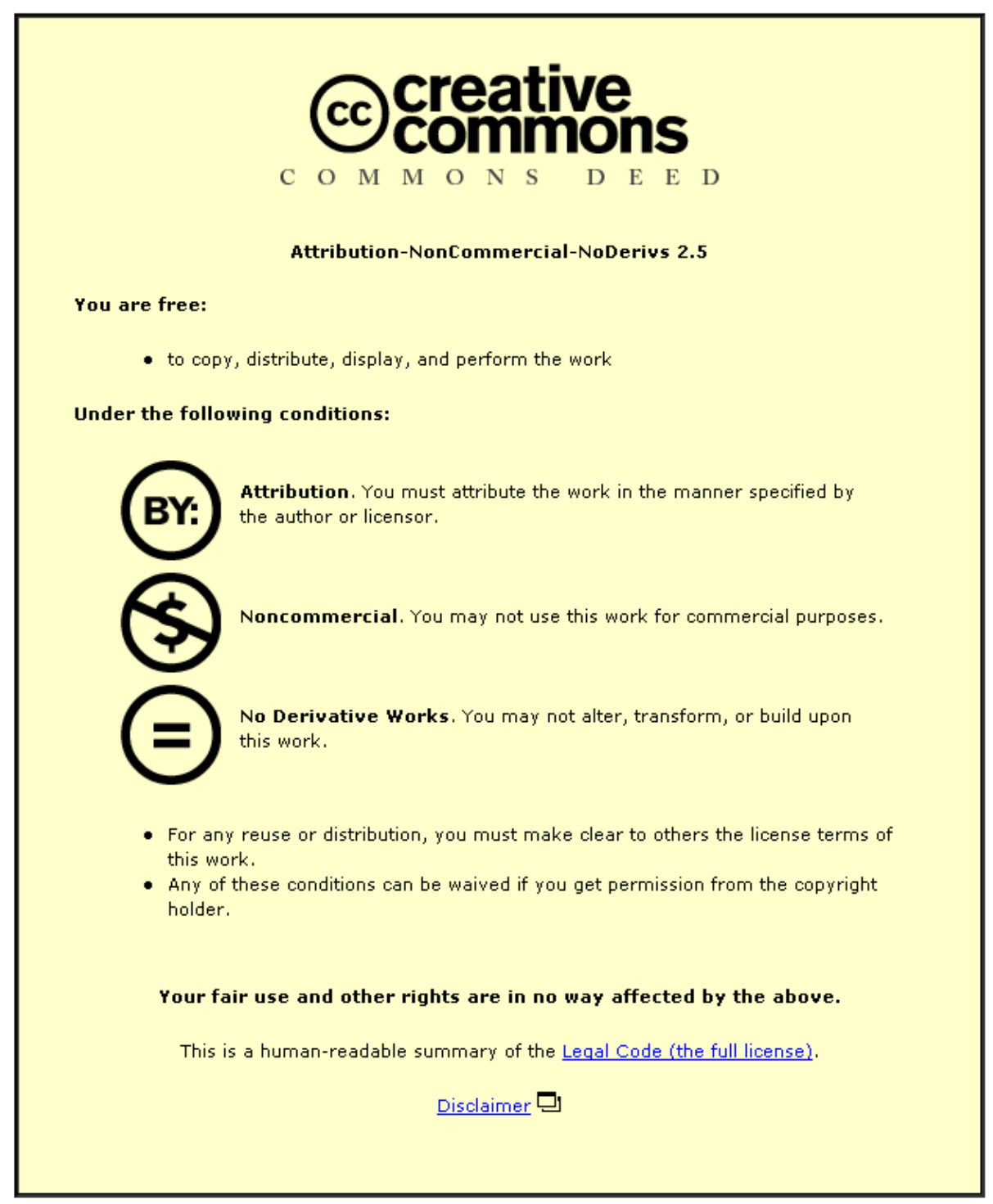

For the full text of this licence, please go to: http://creativecommons.org/licenses/by-nc-nd/2.5/ 


\title{
Microneedles from Fish Scale Biopolymer
}

a Olatunji O*, ${ }^{a}$ Igwe CC, ${ }^{a}$ Ahmed AS, ${ }^{a}$ Alhassan OA, ${ }^{a}$ Asieba GO, ${ }^{b}$ Das DB.

a. Federal Institute of Industrial Research, Oshodi, FIIRO road, Off Agege Motor Road, P.M.B. 21023. Lagos, Nigeria.

b. Chemical Engineering Department. Loughborough University, Loughborough, Leicestershire, LE11 3TU, UK.

* Author for Correspondence (Email: Lolakinola@gmail.com)

\begin{abstract}
This paper reports on microneedles produced from biopolymer films extracted from fish scales of tilapia (Oreochromiss sp) using micromoulding technique. Evaluation of the properties of polypeptide films prepared from the fish scales gave refractive index (1.34), Protein concentration (78\%), ash content (1.6\%) at (22\%) moisture content. The microneedles successfully inserted into artificial skin models and imaging using digital camera showed microneedles remained intact when inserted and when removed from the skin model. Microneedles also successfully inserted into porcine skin and were shown to dissolve gradually at 0 s, 60 s, 120 s and 180 s after insertion. Microneedles containing methylene blue as model drug were also produced and successfully pierced porcine skin. 3D finite element (FEM) simulations were carried out using the measured mechanical properties of the biopolymer films (Young's modulus $0.23 \mathrm{~N} / \mathrm{mm} 2$ and tensile strength $1.8105 \mathrm{~N} / \mathrm{mm} 2$ ) to evaluate the stress distribution on various dimensions of the fish scale derived microneedles and hence, their ability to withstand force necessary to pierce the skin without fracture. Results from mechanical analysis using FEM showed that microneedles with tip radius between 10 and $100 \mu \mathrm{m}$ could withstand up to $0.12 \mathrm{~N}$ of force per microneedle without fracture, which is indicated when the stress at the tip of the microneedle exceeds the ultimate stress of the material of fabrication. Using skin insertion tests and finite element simulations this study provides evidence that microneedles fabricated from fish scale biopolymer can effectively pierce and degrade into skin and therefore are good candidate for transdermal applications.
\end{abstract}

Keywords

Biopolymer, elastomer, modeling, micromoulding, microneedles.

\section{Introduction}

Microneedles have emerged as one of the most effective means of enhancing transdermal penetration of compounds such as proteins, vaccines and therapeutics into the body in a minimally invasive manner (Henry et al, 1998; Prausnitz, 2004; Han and Das, 2013). Microneedles have been fabricated from materials such as silicone, metal and polymer (Davis et al, 2004; Wang et al, 2006; Ito et al, 2006; Olatunji et al, 2013; Nayak and Das, 2013). Biocompatible metals are rather expensive and require more costly processes for microneedle production. On the other hand silicone microneedles have been reported to be relatively brittle (Chen, 2007). while polymer microneedles are a cheaper option that can employ reproducible production methods such as lithography, hot embossing and micromolding (Oh et al, 2008, Park et al, 2005; Lee et al, 2008; Han et al 2007; Donnelly et al 2011). Polymers such as Poly glycolic acid (Park et al, 2006), Polycarbonate (Han et al, 2007) carboxymethyl cellulose (Marin and Andrianov, 2011) and Poly(vinyl methylether-co-melaic acid) (Donelly et al, 2011, Garland et al, 2012) have been used in the fabrication of microneedles in previous studies. Polymer microneedles can be fabricated as solid microneedles which pierce the surface of the skin to create micro conduits through which the compounds to be delivered may permeate the viable epidermis. They can also be designed as 
biodegradable microneedle structures which when inserted into the skin would dissolve in the moist environment of the viable epidermis to release active ingredients incorporated within the microneedle structure (Prausnitz et al, 2004; Garland et al, 2012; Nayak and Das, 2013). This study focuses on the latter i.e. biodegradable polymer microneedles.

More recently the focus in the area of microneedle has been to develop biodegradable and non-toxic polymer microneedles which can be prepared to have sufficiently strong microstructures that can penetrate the skin and deliver active ingredients into the lower layers of the skin and into the body (Nayak and Das 2013). New developments in this area include microneedles produced from rapidly dissolving silk fibrions (You et al, 2011) and trans retinoic acid loaded microneedles for treatment of seborrheic keratosis (Hiraishi et al, 2013). Natural polymers are a preferred choice in contrast to synthetic polymers due to their inexpensive, biodegradable, non toxic nature and availability (Majors and Friedman, 1991). They have been widely used in cosmetics and pharmaceuticals and especially in transdermal drug delivery for controlled release of agents into the body via the skin.

In order for the microneedles to be readily available and affordable, there is a need to explore a wide range of low cost materials for fabrication of biodegradable microneedles. It is also an area of immense interest in all industries to convert wastes to useful end products. This minimizes the cost of waste processing and adds further value to available natural resource.

To this effect, the aim of this study is to explore for the first time, the production of microneedles from biopolymer extracted from fish scales and confirm their ability to penetrate into the skin. Biopolymer extracted from fish scale using thermal hydrolysis dissolves in water, has good film forming ability (Wangtueai and Noomhorm, 2009) and is therefore a good candidate for micromoulding. Fish scales are chosen in particular as they are underutilized natural resource that exists abundantly. Fish scales are the organized outermost structure present on the skin surface of fishes such as tilapia, lizard and grass carp (Chen et al, 2010; Zhang et al, 2011). Furthermore the scales are source of naturally existing protein polymer mainly collagen type 1 . Microstructure analysis of fish scales show collagen fibrils arranged in an orthorgonal plywood structure within a hydroxyappatite matrix (Ikoma et al, 2003; Okada et al, 2011). It is also known that when exposed to water at a temperature of around $80^{\circ} \mathrm{C}$, the collagen from the fish scales is hydrolysed and extracted as collagen polypeptides (Wangtueai and Noomhom, 2009). a more workable form suitable for micromoulding. Recently protein polymers from fish scales in particular collagen and gelatin have gained more interest as an alternative to that from mammalian source due to the fact that unlike that from mammals, fish scale peptides requires a shorter period and less complicated process to extract, eliminates the risk of transferring diseases such as mad cow disease and has some more favorable physical properties such as higher gel strength and gel viscosity (Zhang et al, 2011). Compared to mammalian hydrolyzed collagen, that of fish scale dissolves better at room temperature with better film forming capability (Teramoto et al, 2012).

Delivery of protein biopolymers such as collagen and gelatin to the skin has been shown to have some bioactive properties such as improving skin elasticity, bone strength, antimicrobial activity, mineral binding capacity, lipid lowering effect and immunomodulatory activity (Guillen et al, 2011). In vitro studies on mice have shown that hydrolyzed collagen can act against the skin damage induced by UV radiation (Hou et al, 2009; Zhang and Li, 2009). Protein biopolymers in the form of gelatin 
sponges have also been used for controlling bleeding and healing of surgery wounds (Bing, 1947; Kang et al, 2012). Studies on a group of 63 female Taiwanese subjects aged between 23 and 60 years have also shown that polypeptides extracted from tilapia fish scales improve skin elasticity and moisture content when applied topically (Chai et al, 2010). In the said studies despite the penetration of peptides into the lower layers of the skin, the stratum corneum was found to be a limiting barrier such that the delivery of polypeptides from fish scales to lower layer of the epidermis would prove more effective. Creating microneedles from polypeptides of fish scales therefore has great potential in such applications by further enhancing the penetration of polypeptides into the skin for more effectiveness.

Extraction of biopolymer from fish scale can be carried out using methods such as enzymatic, acid and thermal hydrolysis (Chen et al, 2010, Wangtueai and Noomhorm, 2009; Zhang et al, 2011). The extract is usually a mixture of polypeptides of varying molecular weight, which can be separated by gel chromatography and ultra filtration membrane refining (Chai et al, 2010).

In this paper we report on our work to produce microneedles from polypeptides extracted from fish scales through thermal hydrolysis and evaluate their ability to insert and dissolve into the skin. The microneedles presented are biocompatible microneedles which can either be used to deliver polypeptides to the skin or formulated to incorporate active ingredients such that on insertion into skin will dissolve to release the active ingredients into the skin. For microneedles to be effective in transdermal application either as drug delivery devices or as cosmetic devices, it is important that they are strong enough to penetrate into the skin. The aim of the present paper is to explore the fabrication of novel fish scale biopolymer microneedles and establish their ability to insert and degrade in the skin, thereby determining whether the fish scale biopolymer extracted using thermal hydrolysis is an applicable material for microneedle fabrication in terms of mechanical strength.

\section{Materials and Methods}

Fish scales of tilapia (Oreochromis spp) were obtained from a local fish trader in Ejigbo, Lagos state in Nigeria. The tilapia fish were matured fish of about $1 \mathrm{~kg}$ average weight sourced from the high sea, stored in cold rooms then transported to the retailers. Pig ears were obtained from a butcher at the Odo eran meat market in Lagos state Nigeria. The pigs were slaughtered in the morning, the ears cut off and transported to the laboratory where they were shaved and washed with water. The cartilage and subcutaneous tissue were removed and the skin was washed and stored in the freezer at $-20^{\circ} \mathrm{C}$ until needed for insertion test. Silicone elastomer (Polycraft T4 translucent silicone) was obtained from MBfibreglass, UK and capillary tubes (Pyrex, Stuart, 0.2mm I.D) were obtained from Finlab, Lagos, Nigeria.

\section{Production of Fish scale biopolymer films}

Fish scale biopolymer was extracted according to method reported by Wangtueai and Noomhorm (2009) with slight modifications whereby a centrifuge is used for separation rather than vacuum filtering. The fish scales were washed repeatedly to remove all residue, skin and dirt after which they were kept in the freezer at $-20^{\circ} \mathrm{C}$ until when needed. 100 grams of thawed fish scale was weighed and treated with $0.6 \% \mathrm{w} / \mathrm{v}$ sodium hydroxide concentration in order to remove non collagen residues from the fish scales. Sodium hydroxide pretreatment was carried out at normal room temperature for 1 hour duration while swirling every 15 minutes in a conical flask.

Following pre-treatment, the scales were washed several times using tap water after 
which the sodium hydroxide was completely removed. The scales were then transferred into the stainless steel pressure vessel of 4.5 liters capacity (PC-70001, Binatone, Nigeria) containing $200 \mathrm{ml}$ of water. The vessel was closed and heated at $80^{\circ} \mathrm{C}\left( \pm 3^{\circ} \mathrm{C}\right)$ for 8 hours at a pressure of $80 \mathrm{KPa}$. At these conditions the collagen is hydrolyzed and dissolves in the water (Wahl and Czemuszka, 2006; Zhang et al, 2011). Following extraction the liquid was separated from the solid using a $2 \mathrm{~mm}$ aperture sieve and further separation using a centrifuge (R-8C 3/05 Laboratory centrifuge, REMI Motors, Lagos, Nigeria LTD) at 3500rpm for 20 minutes. Customized silicone plates were prepared using silicone elastomer and activator (Polycraft T4 Silastic, MBFG, Newtownabbey, UK) in the ratio $10: 1$ by weight. The supernatant was transferred unto the customized silicone plate to oven dry at $150^{\circ} \mathrm{C}$ for 1 hour at atmospheric pressure allowing the water to evaporate. The films resulting were then left to air dry on the silicone plate at room temperature for 24 hours after which dry films had been formed. The films were removed from the silicone plates manually and stored at room temperature $\left(30^{\circ} \mathrm{C}\right)$ until next use.

\section{Fabrication of Microneedles}

Microneedles were fabricated using established micromolding techniques (Donnelly et al, 2011; Lee et al, 2008; Park et al, 2006) to fabricate single microneedles from tips of pulled capillary tubes. Glass capillary tubes were pulled on a Bunsen burner to form sharp tips. The tips were characterized using digital imaging to view tips formed. The formed tips were then separated from the rest of the capillary tube by breaking off with tweezers and attached to flat bases of the fish scale polymer film which was slightly wetted to allow adhesion. Silicon elastomer mixed in the ratio 10:1 (weight of silicone to activator) was poured over the glass microneedle master mould and allowed to set for 24hours after which the silicone mould was separated from the glass master mould by hand. Sample of the films were cut with scissors and mixed with a few drops of water to form a thick paste which was then poured unto the silicone mould. The mould filled was then placed in a centrifuge tube and spun at 3500rpm for 20minutes after which it was allowed to air dry at atmospheric temperature and pressure for 24 hours. This was followed by manually separating the microneedle formed from the silicone mould. This is illustrated in figure 1 . Microneedles containing methylene blue as model drug were also fabricated by mixing the wet mould with methylene blue prior to micromoulding. Methylene blue as well as being a dye can be applied as a drug for treatment of idiopathic pruritus ani through intradermal injection and is therefore a good candidate for delivery using microneedle (Sutherland et al, 2008).

\section{Moisture Content Analysis}

The moisture content of the fish scale polymer film was measured using a moisture content analyzer (MS-70, AND, England). 1gram sample of the film was placed on a steel base unto the sample port of the moisture analyzer, the sample was heated at $180^{\circ} \mathrm{C}$ (temperature analysis on the moisture analyzer indicates this as the optimal temperature for the material) until all moisture content is removed from the sample. The value of the moisture content was then read. The moisture analysis was carried out at the same temperature for all film samples used in this study and each sample was determined in triplicate with average value recorded.

\section{Yield}

The yield $(\mathrm{Y})$ was calculated by expressing the mass of the film $\left(\mathrm{M}_{\mathrm{film}}\right)$ as a percentage of the mass of fish scales $\left(M_{\text {scales }}\right)$ used as feedstock. This gives the $\%$ mass of films attainable for every gram of fish scale used. 


$$
Y=\frac{M_{\text {film }}}{M_{\text {scales }}} \times 100 \%
$$

\section{Refractive Index}

The extent to which the polymer produced from fish scales deflected light was measured using a refractometer (60/95 ABBE Type, Bellingham + Stanley Limited, England). $0.1 \mathrm{~g}$ of the film is dissolved in $5 \mathrm{ml}$ of water warmed to $40^{\circ} \mathrm{C}$ and shaken constantly for 1 hour until the films were evenly dissolved in the water. The sample port of the refractometer was wiped using cotton wool prior to pouring in the sample and recording the readings of the refractive index.

\section{Protein Concentration}

In this study we use the Lowry test an dthe nanodrop spectrophotometer (2000/200C. Thermo Scientific, Uk) to confirm the extraction of the biopolymer from the fish scale (Lowry et al, 1951). The Lowry test is used to determine protein concentration based on the presence of pepetide bonds which exist between the amino acids (Lowry et al, 1951). Waterborg et al, 2002; Olson and Markwell, 2007). Number of previous studies have also used the Lowry method to test for hydrolyzed collagen (Komsa-Penkova et al, 1996; Zhou and Regenstein, 2006; Kiew and Don, 2013).

\section{Ash Content}

The ash content of the extracted fish scale biopolymer is obtained using the ASTM D 2974-87 method C (ASTM, 1993). Briefly a porcelain dish was preheated in an oven at $180^{\circ} \mathrm{C}$ for $1 \mathrm{hr}$ and allowed to cool after which the weight was recorded. The dried biopolymer obtained from moisture analysis were added to the crucible and weighed. The porcelain dish is then placed in a furnace and heated to $440^{\circ} \mathrm{C}$ until the sample had completely turned to ash, this took approximately 5 hours. The sample is then removed from the furnace and weighed to obtain the percent ash content.

\section{Mechanical Strength Test}

In order to obtain the mechanical properties of the films which include tensile strength, young modulus, elongation at break and yield strength, the Testometric force analyzer (Testometric, England) was used. The films were cut into equal rectangular pieces of $19 \mathrm{~mm}$ wide. The thickness of the films was obtained by taking average of the thickness of the films at 6 different points along the length using a micrometer. The films were placed between the two grips of the force analyzer and set at an initial distance of $40 \mathrm{~mm}$ between the two grips thus representing the length over which the tensile stress is exerted. At a set speed of $30 \mathrm{~mm} / \mathrm{min}$ the film was pulled in opposite direction by the two grips and a graph of force against displacement was obtained using the software attached to the force analyzer. The surrounding temperature ranged between 25 and $30^{\circ} \mathrm{C}$ over the period of testing.

\section{Insertion Test}

Silicone elastomer (Polycraft T4 Silastic, MBFG, UK) prepared from 10:1 weight ratio of silicone base: activator was used as an artificial skin model. Materials such as silicone have previously been used to mimic the skin in other reported studies (DiMaio and Salcudean, 2005; Guttal et al, 2008). These are used in cases where the skin mechanical properties need to be modeled in a more controlled manner and for more visibility. Single fabricated fish scale microneedles formulated with $0.1 \%(\mathrm{w} / \mathrm{v})$ brilliant red die (Drimarene $\mathrm{X}-2 \mathrm{~B}$, Sandoz) were inserted into the prepared silicone mould under thumb pressure and imaged from the side view. The microneedle was then removed manually at an estimated speed of $5 \mathrm{~mm} / \mathrm{s}$ and examined for any sign of 
fracture. The aim of this experiment was to confirm the physical strength of the microneedles and since the elastomer is moisture free, the microneedle does not dissolve in the artificial skin sample.

Insertion into Porcine Skin

To test the ability of microneedles to pierce real skin, porcine skin was used as model for human skin. Skin insertion experiments were carried out according to method used by Yang et al (2012). Porcine skin were removed from the freezer and allowed to thaw completely at room temperature. The prepared skin was then cut into $3 \mathrm{~cm}$ square area and placed on a ceramic base. The skin was stretched and held in place with the thumb and index finger, microneedles was inserted into the skin and removed immediately after insertion. The points of insertion where stained with methylene blue for 20minutes after which excess methylene blue was wipe off with clean tissue and the insertion points were visualized using digital camera (Nikon, Coolpix L110). To further show the ability of the fish scale microneedles to dissolve in the skin 4 identical microneedles made with the same mould were inserted and removed 0s, 60s, 120s and 180s after insertion into the skin and images of each microneedle taken immediately after removal from skin.

\section{Finite Element Analysis}

Microneedles can be fabricated into various dimensions and the drug delivery rate has been shown to be significantly affected by the geometry of the microneedles such as tip radius (Al-Qallaf and Das, 2008; Olatunji et al 2011). Therefore the fish scale polymer microneedles of various tip sizes must be able to withstand force beyond the force required to penetrate the skin without fracture. Fracture is generally initiated when the stress on the material exceeds the break strength of the material and the maximum stress exists at the tip of the microneedle (Davis et al, 2004). In order to predict the force which the fabricated fish scale biopolymer microneedles of various tip radius can withstand we simulate the stress distribution on the microneedles using the finite element method. 3D images of the prepared microneedles were drafted in AutoCad (Autodesk, 2009). The analysis was carried out using the linear solver independent of time. The images were exported to AutoFEM (AutoFEM Lite ${ }^{\circledR}$, AutoFEM 2013) and solved using the finite element solver which is inbuilt in the software. The software allows 3D simulations of models which have been drafted using AutoCAD to be meshed and solved for mechanical analysis using the FEM. The properties of the material; young modulus and yield stress obtained from experiment were incorporated into AutoFEM, the boundary conditions were defined such that a force was applied at the base of the microneedle and the tips position were fixed. The applied force was varied between $0.03 \mathrm{~N}$ and $0.12 \mathrm{~N}$ per microneedle and the maximum stress at microneedle tips ranging between $10 \mu \mathrm{m}$ and $100 \mu \mathrm{m}$ were recorded. The range for applied force is based on insertion force values reported in literature (Davis et al, 2004; Donelly et al, 2011; Olatunji et al, 2013) and is equivalent to force which can be delivered using thumb pressure. The equivalent stress was calculated from the components of stress as follows:

$$
\sigma_{e q}=\frac{1}{\sqrt{2}} \sqrt{\left(\sigma_{x}-\sigma_{y}\right)^{2}+\left(\sigma_{y}-\sigma_{2}\right)^{2}+\left(\sigma_{z}-\sigma_{x}\right)^{2}+6\left(\tau_{x y}^{2}+\tau_{y z}^{2}+\tau_{x z}^{2}\right)}
$$

Where

$\sigma_{x}=$ normal stress in the direction of the OX-axis of the global coordinate system.

$\sigma_{y}=$ normal stress in the direction of the OY-axis of the global coordinate system.

$\sigma_{z}=$ normal stress in the direction of the OZ-axis of the global coordinate system.

$\mathrm{T}_{\mathrm{xy}}=$ Shear stress acting in the direction of the OY-axis of the global coordinate system 
on a plane with the normal vector parallel to the OX-axis.

$\mathrm{T}_{\mathrm{xz}}=$ Shear stress acting in the direction of the OZ-axis of the global coordinate system on a plane with the normal vector parallel to the OX-axis.

$T_{y z}=$ Shear stress acting in the direction of the OZ-axis of the global coordinate system on a plane with the normal vector parallel to the OY-axis.

\section{Results and Discussion}

Values for the chemical properties of the films; moisture content, protein concentration and ash content alongside refractive index and yield are summarized in table 1 . Tensile strength, young modulus and elongation at break of the fish scale biopolymer films were compared with literature values of two other polymers reported in literature. The polymers compared are 1) a mixture of mammalian biopolymer in the form of hydrolyzed collagen and glycerine (Gel:Gly), this has been studied for use as transdermal patches (Jadhav et al, 2010) and 2) Polymethyl vinyl ether-co-maleic acid crosslinked with polyethyleneglycol (PMVE/MA:PEG), this polymer has been used in microneedle fabrication (Singh et al, 2009; Donelly et al, 2012).

\section{Refractive index}

The refractive index is a basic property of polymers which relates to their ability to bend light, it also correlates with other properties of the polymer such as electromagnetic and chemical properties (Katritzky et al, 1998). Refractive index measured for the fish scale polymer films is within the typical values for polymers where refractive index varies between 1.3 and 1.7. A value of $>1.5$ indicates a high refractive index polymer while values $<1.5$ indicate a low refractive index polymer. The average value for the refractive index of fish scales is 1.34 with a standard deviation of 0.00089 . The fish scale polymer can therefore be classified as a low density polymer.

\section{Moisture Content}

The fish scale polymers retained a level of moisture within their structures. In order to measure the moisture content the films can retain at equilibrium with the surrounding environment, the moisture content was measured again after 4 weeks and 3months, the results are shown in Figure 2. The films maintained moisture content of $18.08 \%$ over the duration of 4 weeks. After another 2 months the moisture contents of the films was analyzed again and found to be $14.27 \%$. Statistical analysis using the student $t$ test gave $p$ value of 0.00135 between $24 \mathrm{hrs}$ and 1 month and $p$ value of 0.00018 between 1 month and 3 months thus showing a reduced level of significance of time with moisture content at 3 months. This can be explained as water molecules binding with the polymers to give them self plasticizing properties such that a level of moisture is maintained in the films at equilibrium. The change in moisture content after 3 months can be attributed to change in seasonal weather condition thereby shifting the equilibium. Previous studies which involved extraction of polymer from fish scales reported moisture content values of about 10.5\% (Wangtueai and Noomhorm, 2009). The difference in moisture content value could be as a result of different conditions and drying methods used. In this study the films were air dried while Wangtueai and Noomhorm (2009) used vacuum drying.

\section{Yield}

The yield for the thermal extraction process was between $6.40 \%$ expressed as percentage of polymer to fish scales on wet basis. Similar studies reported in literature obtained yield values of between 10 and $11 \%$ under optimized conditions for hydrolyzed collagen extracted from lizard fish scales (Wangtueai and Noomhorm, 
2009).

\section{Protein Concentration}

Protein concentration of the polymer films were obtained by Lowry test (Zhou and Regenstein, 2006) using BSA as standard. Protein concentration of $78.2 \%$ was obtained for fish scale biopolymer films extracted in this study. The protein concentration confirms the extraction of proteins from the fish scales and based on the microstructure of fish scales reported in literature (Ikoma et al, 2003; Okada et al, 2011) and the method of extraction used, the polymer can be said to be hydrolyzed collagen. While further analysis on the structure of the polymer extracted is not included in this work, we can confirm extraction of protein polymer from fish scales and study its use for microneedle fabrication.

\section{Ash Content}

In this study, the ash content obtained for biopolymer of tilapia fish scales is $1.60 \%$. This value is below the maximum ash content recommended for edible hydrolyzed collagen which is between $2 \%$ and 2.6\% (Jones, 1977; Haug et al, 2006). Ash content of gelatin from tiger toothed croaker and pink perch bones were $2.7 \%$ and $2.8 \%$ respectively while that recorded for the skin was $0.30 \%$ and $1.88 \%$. For lizard fish scales ash content of about 2.33\% was recorded (Wangtueai and Noomhorm, 2009).

\section{Mechanical Properties of Fish Scale Polymer Films}

The mechanical analysis of the films were carried out for the purpose of obtaining the mechanical properties of the biopolymer produced to be coupled with FEM analysis to test the ability of fish scale microneedles of varying dimensions to withstand force required for the microneedles to insert into the skin.

Table 2 shows results of mechanical properties of the films of fish scale biopolymer (FSBP) compared with literature values of mechanical properties of mammalian biopolymer (hydrolyzed collagen) films plasticized with glycerine (GEL:Gly) and films of poly(methylvinylether/maelic anhydride) crosslinked with polyethyleneglycol plasticizer (PMVE/MA:PEG). These two polymers have been used for transdermal drug delivery applications in other studies. PMVE/MA:PEG films in particular have been used for microneedle fabrication (Donelly et al, 2012). Compared to previous studies which reported hydrolyzed collagen films from mammalian source containing no plasticizer to be brittle and dry with no flexibility (Jadhav et al, 2010), the fish scale polymer films from this study are strong and flexible. Films of hydrolyzed collagen and glycerine (GEL:Gly) in the ratio $20: 5$ polymer had elongation of $71.65 \%$ while that of fish scale polymer obtained in this study was up to 5 times higher at $393.45 \%$ with a standard deviation (SD) of 227.54. Similarly the tensile stress and young modulus were also much higher. The films of 15\% PMMVE/MA crosslinked with PEG 10,000 in the ratio 2:1 showed tensile strength and young modulus which were 91 times and 17 times higher than that of fish scale polymer $(p=0.0092,, p=0.064$ respectively). However the fish scale polymer had similar (1.26 times higher) elongation at break than the PMVE/MA films compared $(p=0.66)$. Values for films of PMVE/MA without plasticizer which have been used for microneedle fabrication were not determined (Singh et al, 2009; Donelly et al, 2011). From these results it is observed that values for the mechanical properties of fish scale polymers obtained here vary from that of polymer films which have been studied for microneedle fabrication. However these results are not conclusive, further analysis are discussed in subsequent sections to determine the suitability of the polypeptides obtained from fish scales for microneedle fabrication. 


\section{Microneedle Fabrication}

Microfabrication of microneedles using pulled glass capillary tubes and silicone micromoulding produced tapered microneedles of about $900 \mu \mathrm{m}$ in length as shown in Figure 3 and 4 . The fish scale polymer formed into microneedles geometries replicating the structure of the glass microneedles used as master moulds. Figure $3 a$ shows microneedles fabricated with plain fish scale polymer while figure $3 b$ shows fish scale microneedles containing $1 \% \mathrm{w} / \mathrm{v}$ methylene blue. This results show that fish scale polymers can be used to produce microstructures suitable for microneedle application using reproducible micromoulding techniques. It also shows that the fish scale polymer microneedles can incorporate model drug such as methylene blue and by visual inspection we see that methylene blue was evenly spread across the length of the microneedles. The structures obtained are comparable with silk fibrion microneedles containing methylene blue which were produced by You et al (2011). In the present study the glass capillary tubes were pulled by hand over a Bunsen burner to obtain microneedle at the tips as described in the method. However for mass production sharp tips can be reproducibly produced using micropipette puller as reported in previous studies. For example Wang et al (2006) have used glass microneedles pulled using automated micropipette puller for microinjection and as master moulds for fabricating polymer microneedles. The group obtained similar structures with tips of about $100 \mu \mathrm{m}$.

\section{Insertion into Artificial Skin Model}

To analyze the strength of the microneedles produced, we insert into a skin phantom made from silicone. Silicone has similar flexibility as the skin with a young modulus of $\sim 0.916 \mathrm{MPa}$ (Peel and Jensen, 2000) and it has been widely used to model the skin as it has similar physical properties to the skin in terms of toughness and flexibility (Guttal et al, 2008), and can therefore be used as a model to test the ability of microneedles fabricated in this study to insert into skin without failure. Figure 4 shows microneedle while inserted and following removal from artificial skin model, microneedles remained intact while inserted into the skin model and upon removal without fracture. The results show that fish scale microneedles possess sufficient strength to successfully insert into skin model without breakage or fracture.

\section{Insertion into Porcine Skin}

Figure 5 shows points where different microneedles were inserted into porcine skin followed by methylene blue staining. Both plain microneedles and microneedles containing methylene blue successfully pierced the skin. To further show the ability of the fish scale microneedles to degrade in the skin 4 different microneedles were inserted and removed 0s, 60s, 120s and 180s after insertion into the skin. Figure 6 shows images of microneedles gradually dissolving into the skin. The microneedles dissolved completely after 180 seconds insertion time (Figure 6d). These microneedles take longer to dissolve compared to the silk fibrion microneedles which completely dissolve in 60 seconds in porcine skin (You et al, 2011).

\section{FEM modeling}

To further study the effect of applied load on the microneedles, we model microneedles with dimensions as reported in literature (e.g. Donelly et al, 2010, Olatunji et al, 2011). The microneedles modeled were $600 \mu \mathrm{m}$ long with a base radius of $150 \mu \mathrm{m}$ and tip radius ranging between $10 \mu \mathrm{m}$ and $100 \mu \mathrm{m}$. Polymer microneedles of similar dimensions have been previously shown to withstand up to $0.03 \mathrm{~N}$ force per microneedle which was 
necessary for insertion into porcine skin models (Donelly et al, 2010). Therefore the microneedles fabricated in this study must be able to withstand such force in order to be fit to penetrate into the skin. The stress at tip for force ranging between $0.03 \mathrm{~N}$ to $0.12 \mathrm{~N}$ is modeled as shown in figure 5 . The results in figure 6 show that the single fish scale microneedles with tip radius ranging between $10 \mu \mathrm{m}$ and $100 \mu \mathrm{m}$ can withstand up to $0.12 \mathrm{~N}$ force without fracture since for all the simulations, the stress at tip of the microneedles did not exceed the ultimate stress of the fish scale polymer material. The stress was most concentrated at the tip of the microneedles in accordance with other reports (Davis et al, 2004; Roxhed et al, 2007) where it has been reported that microneedles were more likely to fracture at the tip. Finite element modeling has been previously applied in similar manner in biomedical research in for example bone fracture prediction (MacNeil et al, 2012) and also predicting optimal drug release from microneedle structures (Al-Qallaf and Das, 2008; Olatunji et al, 2012), insertion force optimization of microneedles (Olatunji et al, 2013). The results from these simulations provide evidence that fish scale polymer microneedles can be fabricated into a range of dimensions and maintain sufficient mechanical strength to pierce the skin.

From the results of the experiment and FEM analysis it seems that despite having much lower tensile strength compared to PMVE/MA formulation which has also been used in microneedle fabrication (Donelly et al, 2012; Donelly et al, 2010; Singh et al, 2009), the fish scale polymer microneedles can successfully pierce into skin as shown in the insertion test for artificial and porcine skin. This is understandable as the microneedles fabricated with PMVE/MA have indeed been shown to withstand up to $0.71 \mathrm{~N}$ of force per microneedle, forces much higher than the force required to pierce into porcine skin, which is about $0.03 \mathrm{~N}$ per microneedle (Donelly et al, 2010).

This study introduces microneedles from fish scale biopolymer which demonstrate sufficient mechanical strength to pierce skin models without additional polymer incorporated thereby decreasing cost and complexity of production. There have been some previous studies reporting the fabrication of microneedles from biodegradable polymer. However this is the first time production of microneedles from polymer extracted from fish scales, an aquatic waste material, is being presented. The microneedles produced in this study demonstrate sufficient strength to penetrate skin model without the need for additional component such as copolymer or plasticizer unlike the microneedles fabricated from the other polymers incorporating additional component such as poly lactic acid (PLA), Poly glycolic acid (PGA), PVP and PVA (Wang and Jeng, 2009; Kim et al 2012); Ke et al, 2012; Chu et al, 2010; Nayak and Das, 2013) in order to achieve sufficient mechanical strength. The fish scale biopolymer is a natural protein which is potentially biocompatible and is therefore an ideal candidate for microneedle production.

\section{Conclusion}

This study presents biodegradable polymer microneedles fabricated from biopolymer extracted from fish scales. Microneedles were successfully produced from the fish scale polymer films using reproducible micromoulding techniques. The microneedles fabricated were shown to have sufficient mechanical strength to pierce into artificial skin model without fracture and they were further shown to pierce and dissolve into porcine skin without use of additional plasticizer or copolymer. This study explores a cheaply available raw material for the production of biodegradable polymer microneedles. In addition to their use as delivery systems for drugs, these microneedles have great potential for cosmetic application and general health benefits 
even in their plain form due to the intrinsic bioactivities of the fish scale biopolymer used as material of fabrication.

\section{Acknowledgement}

The authors would like to acknowledge the support of Dr G.N Elemo, director general of the federal institute of industrial research Oshodi on this project.

\section{References}

1. Al-Qallaf B., Das D.B., (2008) Optimization of Square Microneedle Arrays for Increasing Drug Permeability in Skin. Chem. Eng. Sci. 63:2523-2535.

2. ASTM Standard D 2974-87, (1993) "Standard Test Method for moisture, ash and organic matter of peat and other organic soils" Annual Book of ASTM standards. Philadelphia PA 19103. 31-33.

3. Bing j. (1947) Experimental observations on the use of a Danish gelatin sponge preparation (Spongostan) as an absorbable haemostatic agent. Acta Pharmarcol Toxicol (Copenh) 3:3564-372.

4. Chai H, Li J, Huang H, Li T, Chan Y, Shiau C, Wu C. (2010) Effects of Sizes and Conformations of Fish scale collagen peptides on facial skin Qualities and Transdermal Penetration Efficiency. Journal of Biomedicine and Biotechnology. 2010(757301):1-9

5. Chen B., Wei J., Tay F.E.H., Wong Y.T., Iliescu C., (2007) Silicon Microneedle array with Biodegradable Tips for Transdermal drug delivery. DTIP of MEMS and MOEMS. ISBN 978-2-35500-000-3.

3. Chen S., Hirota N., Okuda M., Takeguchi M., Kobayashi H., Hanagata N., Ikoma T., (2010) Microstructures and rheological properties of Tilapia fish-scale collagen hydrogels with aligned fibrils Fabricated under Magnetic Fields. Acta Biomaterialia. 7:644-652.

4. Chu LY., Choi SO., Prausnitz MR., (2010) Fabrication of Dissolving Polymer Microneedles for Controlled Drug encapsulation and Delivery: Bubble and Pedestal Microneedle Designs. J.Pharm Sci. 99:4228-4238.

5. Davis, S.P, Landis, B.J, Adams, Z.H, Allen, M.G, Prausnitz, M.R. (2004). Insertion of microneedles into skin: measurement and prediction of insertion force and needle fracture force. J Biomechanics. 37, 1155-1163

6. DiMaio SP, Salcudean SE. (2005) Interactive simulation of needle insertion models. IEEE Transactions in Biomedical Engineering;52(7):1167-1179.

7. Donnelly RF, Garland MJ, Morrow DIJ, Migalska K, Singh TR, Majithiya R, Woolfson AD (2010). Optical coherence tomography is a valuable tool in the study of the effects of microneedle geometry on skin penetration characteristics and in-skin dissolution J. Cont. Rel. 147, 333-341

8. Donnelly RF, Majithiya R, Singh TRR, Morrow DIJ, Garland MJ, Demir YK, Migalska K, Ryan E, Gillen D, Scott CJ, Woolfson DA. (2011). Design, optimization and characterisation of polymeric microneedle arrays prepared by a novel laser-based micromoulding technique. Pharm. Res. 28, 41-57

9. Donelly R.F, Singh T.R.R, Garland M.J, Migalska K, Majithiya R, McCrudden C.M, Kole P.L, Mahmood T.T.M, McCarthy H.O, Woolfson D. (2012) HydrogelForming Microneedle Arrays for Enhanced Transdermal Drug Delivery. Adv. Funct Mat. 22(23):4879-4890.

10. Garland MJ., Migalska K., Tuan-Mahmood TM., Singh TRR., Majithija R., Caffarel-Salvador E., McCarthy HO., Woolfson AD., Donnelly AF (2012) Influence of Skin Model on in vitro Performance of Drug-loaded Soluble Microneedle arrays. Int. J. Pharm. 434:80-89. 
10. Guttal S.S., Patil N.P., Nadiger R.K., Kulkarni R. (2008) A Study on Reproducing Silicone Shade Guide for Maxillofacial Prostheses Matching Indian Skin Color. Ind. J. Dent. Res. 19(3): 191-195.

11. Gomez-Guillen MC, Gimenez B, Lopez-Caballero ME, Montero MP (2011) Functional and Bioactive Properties of Collagen and Gelatin form Alternative Sources: A Review. Food Hydrocolloids. 25(8): 1813-1827.

12. Han M., Hyun D., Park H., Lee S.S., Kim C., Kim C.G., (2007) A novel Fabrication Process for Out-of-plane Microneedles Sheets of biocompatible polymer. J. Micromech. Microeng. 17: 1184-1191.

13. Han T., Das D.B (2013) Permeability Enhancement for Transdermal Delivery of Large Molecules. J. Pharm. Sci. 102(10):355614-3622. DOI: 10.1002/jps.23662.

13. Haug I.G, Draget K.I, Smidsrod O. (2004) Physical and Rheological Properties of Fish Gelatine Compared to Mammalian Gelatin. Foods Hydrocolloids. 18;203-213.

14. Henry S, McAllister DV, Allen MG, (1998). Prausnitz MR. Microfabricated microneedles: a novel approach to transdermal drug delivery. J. Pharm. Sci. 87, 922-925.

14. Hiraishi Y., Hirobe S., lioka H., Quan Y.S., Kamiyama F., Asada H., Okada N., Nakagawa S., (2013) J. Control Release. 171(2): 93-103.

15. Ito Y, Jun-Ichiro Y., Keiji S, Nobuyuki S., Kanji T., (2006) Self-dissolving microneedles for the percutaneous absorption of EPO in mice. $J$ Drug Target. 14:255-261.

16. Ito Y., Eiji H., Atsushi S., Nobuyuki S., Kanji T., (2006) Feasibility of Microneedles for percutaneous Absorption of Insulin. Eur. J. Pharm. Sci. 29:8288.

17. Jadhav R.T., Kasture P.V., Gattani S.G., Surana S.J., (2010) Formulation and Evaluation of transdermal films of Diclofenac Sodium. Int. J Chem Tech Res. 2(1): 354-360.

18. Jones NR (1977) Uses of gelatin in edible products. In: Ward AG, Courts A. (Eds) The Scince and Technology of Gelatin. Accademic Press London. Pp.336394.

19. Ke CJ., Lin YJ., Hu YC., Chiang WL., Chen KJ., Yang WC., Liu HL., Fu CC., Sung HW., (2012) Multidrug Release Based on Microneedle Arrays Filled with pH-responsive PLGA microsphere. Biomaterials. 33:5156-5165.

20. Kiew and Don (2013) Modified Lowry's Method for Acid and Pepsin Soluble Collagen Measurement from Clarias Speies Muscles. Open Acess Scientific Reports. 2(3):1-5.

21. Kim MY., Jung B., Park JH., (2012) Hydrogel swelling as a trigger to release biodegradable Polymer Microneedles in Skin. Biomaterials. 33:668-678.

20. Koli MJ, Subrata B, Nayak BB, Patange SB, Pagarkar AU, Gudipati V. (2012) Functional characteristics of gelatin extracted from skin and bones of tiger toothed croaker (Otolithes ruber) and pink perch (Nemipterus Japonicus). Food and Bioproducts Processing. 90:555-562.

21. Komsa-Penkova R, Spirova R, Bechev B. (1996) Modification of Lowry's method for Collagen Concentration Measurment. J. Biochem BioPhys Methods. 32(1):33-43.

22. Ktaritzky A.R., Sild S., Karelson M. (1998) Correlation and prediction of the 
refractive indices of Polymers by QSPR. J. Chem Inf. Comput Sci. 38(6): 11711176.

23. Lee J.W., Park J.H., Prausnitz M.R. (2008) Dissolving microneedles for transdermal drug delivery. Biomaterials. 29: 2113-2124.

24. Lee $\mathrm{K}$, Lee $\mathrm{CY}$, Jung $\mathrm{H}$. (2011) Dissolving microneedles for transdermal drug administration prepared by stepwise controlled drawing of maltose. Biomaterials. 32(11): 3134-3140.

25. Lowry OH, Rosebrough NJ, Farr AL, Randall RJ (1951) Protein measurement with Folin phenol reagent. J Biol Chem 193:265-275.

26. MacNeil J.A., Adachi J.D., Goltzman D., Josse R.G., Kovacs C.S., Prior J.C., Olszynski W., Davison K.S., Kaiser S.M. (2012) Predicting fracture using 2D Finite element Modelling. J. Med. Eng. Phys. 34(4):478-484.

24. Majors K.R., Friedman M.B. (1991) Animal testing of Polymer based Systems. In TarchaPJ, editor. Polymers for Controlled Drug Delivery. Boca Raton, FL, usa: CRC Press Inc., 1991, 231-239.

25. Marin A., Andrianov A.K., (2011) Carboxymethylcellulose-Chitosan-coated microneedles with Modulated Hydration Properties. J. Appl. Polym. Sci. 121(1):394-401.

26. Nayak A., Das D.B., (2013) Potential of Biodegradable Microneedles as a Transdermal Delivery Vehicle for Lidocaine. Biotechnol Lett. 35:1351-1363.

27. Oh J.H., Park H.H., Do K.Y., (2008) Influence of the delivery systems using a microneedle array on the permeation of a hydrophilic molecule calcein. Eur. J. Pharm. Biopharm 68:1040-1045.

28. Okuda M, Ogawa N, Takeguchi M, Hashimoto A, Tagaya M, Chen S, Hanagata $\mathrm{N}$, Ikoma T. (2011) Minerals and aligned collagen fibrils in tilapia fih scales: Structural analysis using dak-field and energy-filtered transmission electron microscopy and electron tomography. Microc Microanal. 17(55): 788-798.

29. Olatunji O, Das D.B, Garland M.J, Belaid L, Donnelly R.F. (2013) Influence of Array Interspacing on the force required for successful microneedle Skin Penetration: Theoretical and Practical Approaches. J. Pharm. Sci. 102(4): 12091221.

30. Olatunji O, Das D.B, Nassehi V. (2012) Modeling Transdermal Drug Delivery Using Microneedles: Effect of geometry on Drug Transport Behaviour. J. Pharm. Sci. 101(1):164-175.

30. Olson BJSC, Maxwell J. (2007) Assay for determination of protein concentration. In current Protocols in Protein science. John Wiley and Sons Inc. USA.

31. Park J., Allen M.G., Prausnitz M.R., (2006) Polymer Microneedles for Controlled-Release Drug Delivery. Pharm. Res. 23(5):1008-1019.

32. Park J.H., Allen M.G., Prausnitz M.R., (2005) Biodegradable polymer microneedles: fabrication and transdermal drug delivery. J. Control Release. 104: 51-66.

32. Peel LD., Jensen DW., (2000) The Response of Fibre Reinforced Elastomer Under Simple Tension. Journal of Composite Materials. 34: 1-86.

33. Prausnitz MR. (2004). Microneedles for transdermal drug delivery. Advanced Drug Delivery Review. 56, 581-587

34. Roxhed N., Gasser C.T, Griss P. (2007) Penetration-Enhanced Ultrasharp 
Microneedles and Prediction on Skin Interaction for Efficient Transdermal Drug Delivery. J. MEMS. 16(6):1429-1440.

35. Singh T.R., McCarron P.A., Woolfson D.A., Donnelly R.F. (2009) Physicochemical Characterization of Poly(ethylene glycol) plasticized Poly(methy vinyl ether-co-maleic acid) Films. J. Appl. Polym. Sci. 112: 27922799.

36. Sutherland AD, Faragher IG, Frizelle FA. (2008). Intradermal Injection of Methylene blue for the treatment of refractory pruritus ani. Colorectal Dis. 11(3): 282-287.

37. Teramote N, Hayashi A, Yamamaka K, Sakiyama A, Nakano A, Shibata M. (2012) Preparation and Mechanical Properties of Photo-Crosslinked Fish Gelatin/Imogolite Nanofiber Composite Hydrogel. Materials. 5:2573-2585.

38. Van Krevelen D.W. (1972) Properties of Polymers: Correlation with Chemical Structure; Elselvier: Amsterdam; Chapter 11, p195.

37. Wahl and Czermuszka, Collagen-hydroxyappatite composition for hard tissue repair. European Cells and Materials. 11: 43-56.

38. Wang MW., Jeng H., (2009) Optimal moulding parameter design of PLA micro lancet Needles Using the Taguchi Method. Polym. Plast. Technol. 48:730-735.

39. Wang P.M., Cornwell M., Hill J, Prausnitz M.R. (2006) Precise microinjection into skin using hollow microneedles. J. Invest. Dermatol. 126:1080-1087.

40. Wangtueai,S., Noomhorm, A., (2009). Processing optimization and characterization of gelatin from lizardfish (Saurida spp.) scales. LWT- Food Science and Technology. 42: 825-834.

41. Waterborg JH, Mathews HR (2002) The Lowry method for protein quantitation. In: The Protein Handbook. (2nd edn), Humana Press, New Jersey.

42. Yang S., Feng Y., Zhang L, Chen N, Yuan W, Jin T (2012) A scalable fabrication Proces of polymer microneedles . International Journal of Nanomedicine. 7:1415-1422

41. You, X., Chang, J., Ju, B., Pak, J. (2011).Rapidly dissolving fibroin microneedles for transdermal drug delivery. Mat. Sci. Eng C. 31(8): 1632-1636.

42. Zhang F., Xu S., Wang Z. (2011) Pre-treatment Optimization and Properties of Gelatin from Freshwater Fish Scales. Food and Bioproducts Processing. 89:185-193.

43. Zhou P, Regenstein JM. (2006) Determination of Total Protein Content in Gelatin Solutions withLowry or Biuret Assay. J. food Sci. 17(8):C474-C479.

44. Pati R., Adhikan B, Dhara S. (2010) Isolation and Characterization of Fish Scale Collagen of Higher Thermal Stability. Bioresource Technology. 101: 3737-3742. 
List of Tables

Table 1

Table 2

\section{List of Figures}

Figure 1

Figure 2

Figure 3

Figure 4

Figure 5

Figure 6

Figure 7

Figure 8
Refractive index, yield, ash content, moisture content and Protein concentration of produced films.

Mechanical properties of the fish scale polymer films compared with PMVE/MA and Gel/Gly films.

Schematics of microneedle fabrication.

Moisture content of films 24hrs, 4 weeks and 3 months after air drying.

Digital image of microneedles fabricated from fish scale

Digital image of microneedles inserted into artificial skin model and after removal from skin model

Porcine Skin stained with methylene blue with arrows showing Microneedle insertion points

Microneedles removed from porcine skin after a) 0s b) 60s c)120s and d)180s

Microneedles with tip radius of $10 \mu \mathrm{m}$ with $0.06 \mathrm{~N}$ evenly distributed force applied.

Stress at tip of microneedles when force of $0.03-0.12 \mathrm{~N}$ is applied. 
Table 1 Properties of produced films.

\begin{tabular}{|l|l|}
\hline Property & Value \\
\hline Moisture Content (\% wet basis) & $22.16 \pm 0.51$ \\
Protein concentration (\% wet basis) & $78.20 \pm 0.53$ \\
Ash content (\% wet basis) & $0.10 \pm 0.19$ \\
Refractive index (-) & $1.34 \pm 0.00$ \\
Yield (\%, wet basis) & $6.40 \pm 1.02$ \\
\hline
\end{tabular}

\pm standard deviation 
Table 2 Mechanical properties of the fish scale polymer (FSBP) films compared with other polymer films.

\begin{tabular}{|l|l|l|l|}
\hline & $\begin{array}{l}\text { Young modulus } \\
\left(\mathrm{N} / \mathrm{mm}^{2}\right)\end{array}$ & $\begin{array}{l}\text { Ultimate stress } \\
\left(\mathrm{N} / \mathrm{mm}^{2}\right) \\
(\%)\end{array}$ \\
\hline FSBP & $0.2324 \pm 0.028$ & $1.8105 \pm 0.33$ & $393.45 \pm 41.04$ \\
\hline${ }^{*}$ Gel:Gly 20:5 & 1.452 & 1.040 & 71.66 \\
\hline$\star *$ PMVE/MA:PEG 2:1 & $21.19 \pm 6.89$ & $31.60 \pm 7.89$ & $311.99 \pm 116.12$ \\
\hline
\end{tabular}

*Values taken from Chai et al (2010), **values taken from Singh et al (2009) +standard deviation 


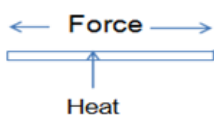

Heat

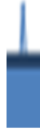

The tip is attached

unto a base with

adhesive

Microstructure is

copied unto silicone

mould

Microneedle is formed upon

drying and separation from

mould.
Tapered tip is

formed

\section{Silicone mould is}

poured over the

glass master mould

Polymer is poured into

mould, centrifuged and

dried

Figure 1. Schematics of microneedle fabrication. 


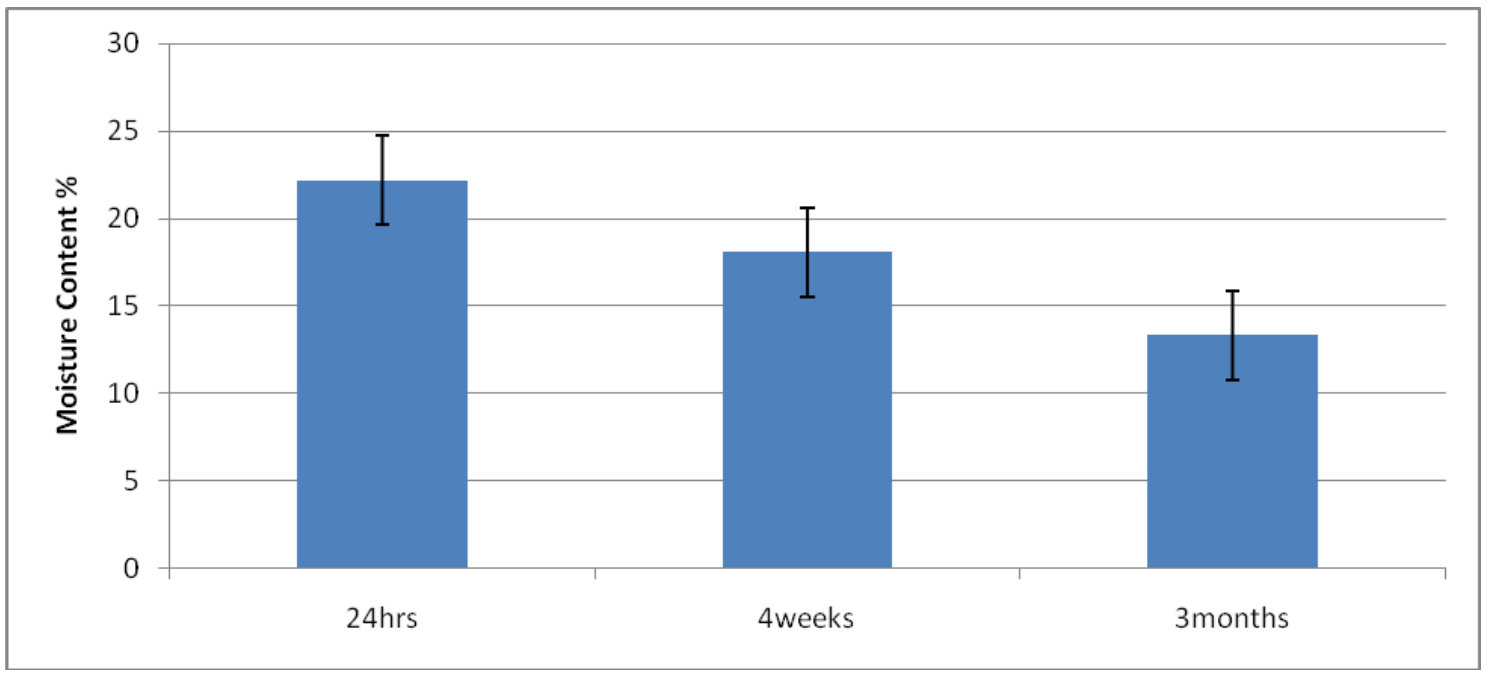

Figure 2. Moisture content of films $24 \mathrm{hrs}, 4$ weeks and 3 months after air drying. 


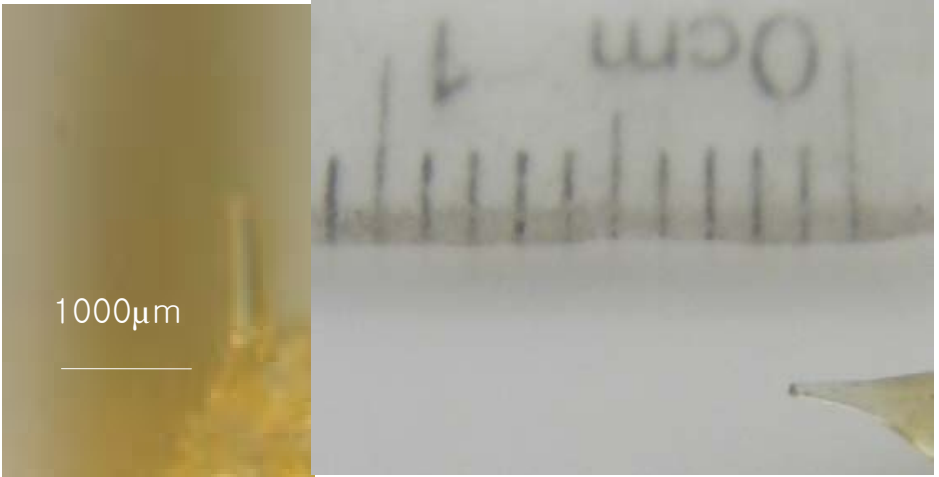

1)

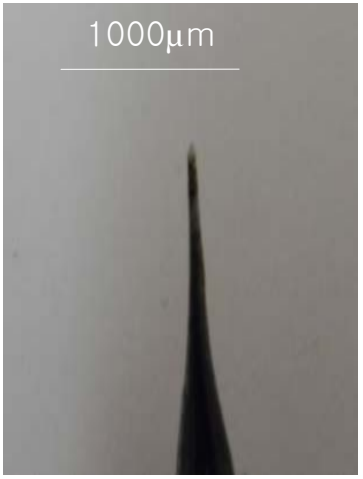

b)

Figure 3 digital image of microneedles fabricated from fish scale a) Plain and b) containing methylene blue 

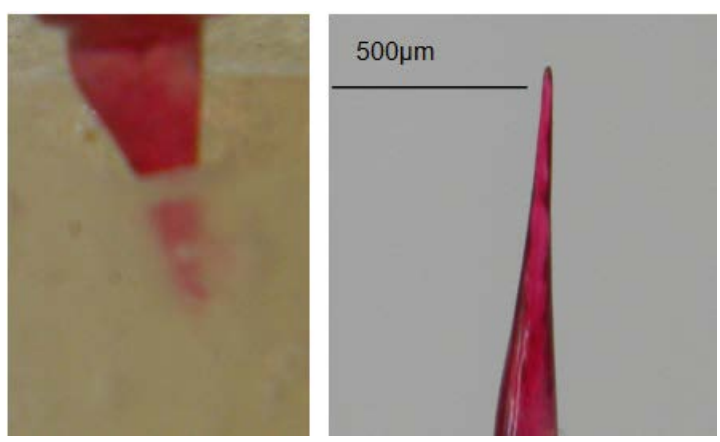

Figure 4 digital image of microneedles inserted into artificial skin model and after removal from skin model 


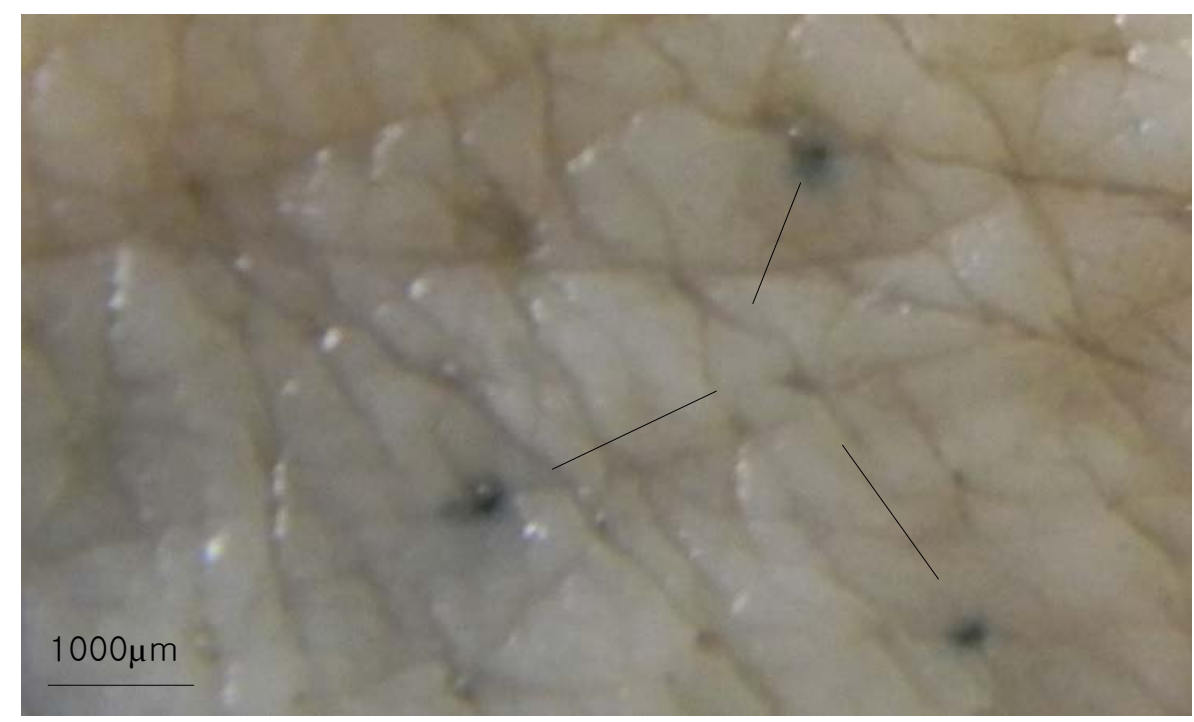

Figure 5. Porcine Skin stained with methylene blue with arrows showing Microneedle insertion points. 

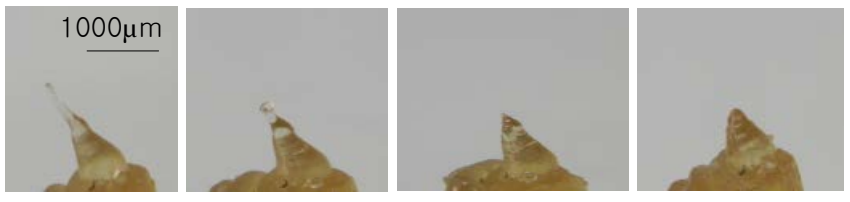

Figure 6. Microneedles removed from porcine skin after a) 0s b) 60s c)120s and d)180s (same scale for all images). 


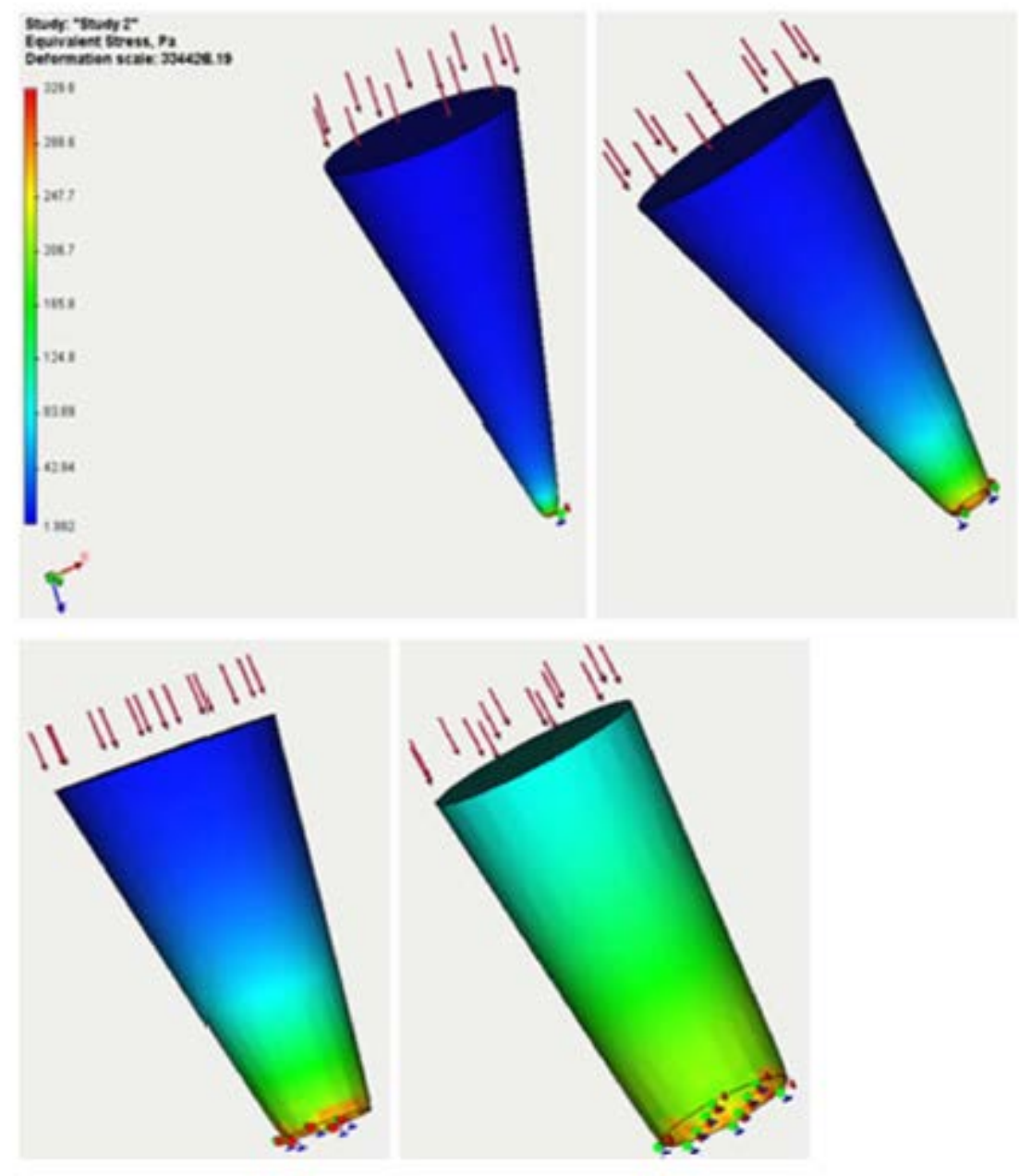

Figure 7. Microneedles with tip radius of $10 \mu \mathrm{m}$ with $0.06 \mathrm{~N}$ evenly distributed force applied 


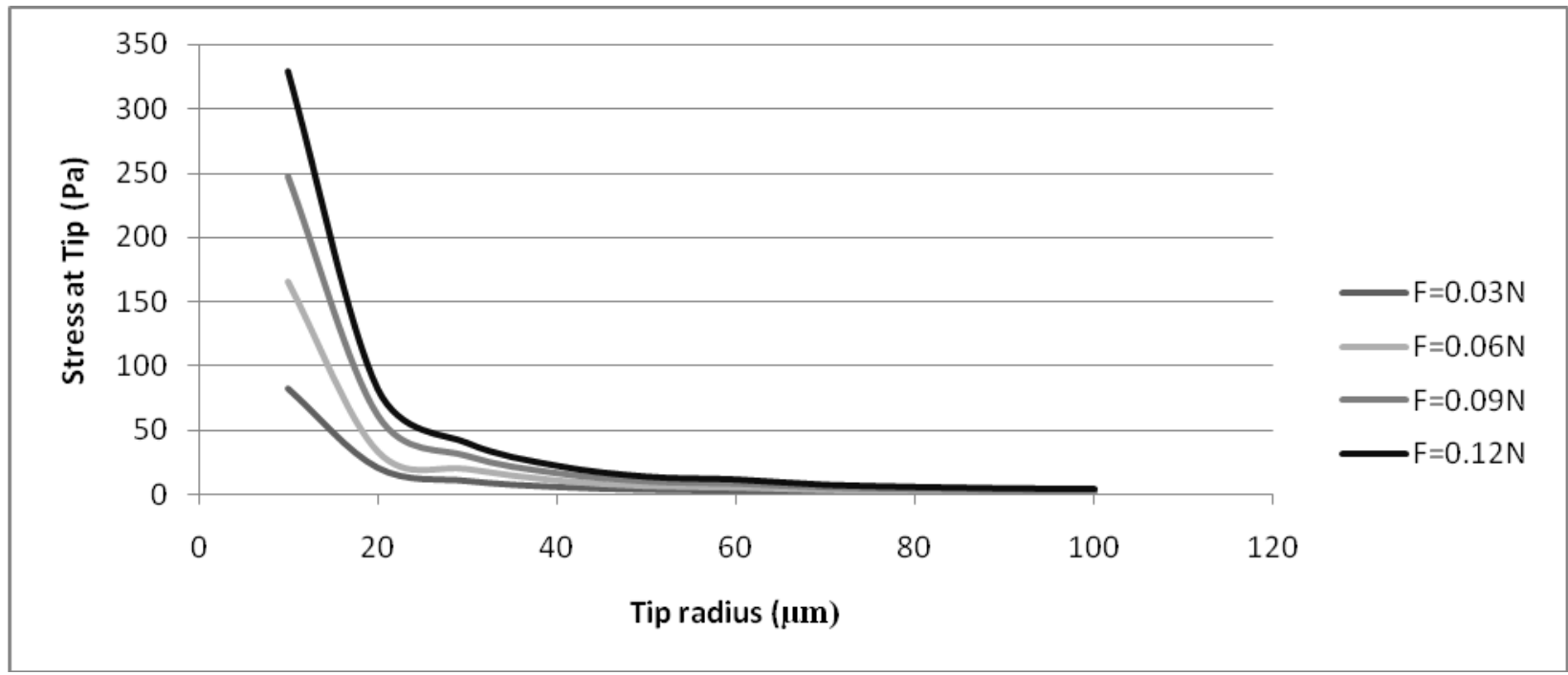

Figure 8. Stress at tip of microneedles when force of $0.03-0.12 \mathrm{~N}$ is applied. 